University of Nebraska - Lincoln

DigitalCommons@University of Nebraska - Lincoln

Publications, Agencies and Staff of the U.S.

Department of Commerce

U.S. Department of Commerce

2008

\title{
An animal movement model incorporating home range and habitat selection
}

Aaron Christ

Alaska Department of Fish and Game

Jay Ver Hoef

National Marine Mammal Laboratory

Dale L. Zimmerman

University of lowa

Follow this and additional works at: https://digitalcommons.unl.edu/usdeptcommercepub

Part of the Environmental Sciences Commons

Christ, Aaron; Ver Hoef, Jay; and Zimmerman, Dale L., "An animal movement model incorporating home range and habitat selection" (2008). Publications, Agencies and Staff of the U.S. Department of Commerce. 184.

https://digitalcommons.unl.edu/usdeptcommercepub/184

This Article is brought to you for free and open access by the U.S. Department of Commerce at DigitalCommons@University of Nebraska - Lincoln. It has been accepted for inclusion in Publications, Agencies and Staff of the U.S. Department of Commerce by an authorized administrator of DigitalCommons@University of Nebraska - Lincoln. 


\title{
An animal movement model incorporating home range and habitat selection
}

\author{
Aaron Christ · Jay Ver Hoef • Dale L. Zimmerman
}

Received: 1 August 2005 / Revised: 7 July 2006 / Published online: 19 September 2007

(C) Springer Science+Business Media, LLC 2007

\begin{abstract}
Wildlife biologists are often interested in how an animal uses space and the habitat resources within that space. We propose a single model that estimates an animal's home range and habitat selection parameters within that range while accounting for the inherent autocorrelation in frequently sampled telemetry data. The model is applied to brown bear telemetry data in southeast Alaska.
\end{abstract}

Keywords Brown bear - GPS telemetry $\cdot$ Markov chain Monte Carlo $\cdot$ Resource selection function · Utilization distribution · Vector autoregression

\section{Introduction}

How an animal uses space and how it uses the habitat resources within that space are two common questions in wildlife biology. One major factor influencing the path an animal follows is the need for certain resources along that path. These resources may fill requirements

This article is based on a portion of this author's Ph.D. dissertation completed in 2003 at the University of Iowa.

\section{A. Christ}

Alaska Department of Fish and Game, 333 Raspberry Road, Anchorage, AK 99518, USA

e-mail: aaron_christ@fishgame.state.ak.us

J. Ver Hoef

National Marine Mammal Laboratory, 7600 Sand Point Way NE Bldg 4, Seattle, WA 98115-6349, USA

e-mail: jay.verhoef@noaa.gov

D. L. Zimmerman ( $\varangle)$

Department of Statistics and Actuarial Science, University of Iowa, 241 Schaeffer Hall, Iowa City, IA 52242, USA

e-mail: dzimmer@stat.uiowa.edu 
for nutrition, protection from predation, reproduction, etc. In this article we develop a unified model for telemetry relocation data that accounts for both movement and the use of resources implied by that movement.

Telemetry data may be used to model movement both directly and indirectly. The direct approach is to model the instantaneous trajectory vector of the animal, which is approximated by differencing each consecutive pair of observed locations. What results is a model of the velocity field, which describes the strength of attraction toward (or aversion from) various regions in space. This is the diffusion modeling approach taken by Brillinger (1997) and Brillinger et al. (2002, 2004). However, the suitability of this approach requires that the relocations are taken at a sufficiently fine time resolution to yield a good approximation to the instantaneous trajectory vector. For migratory movement, the trajectory vector may be relatively stable over time. However, for large terrestrial mammals that exhibit fidelity to a "home range," the time resolution of the data often is too crude to support diffusion-type models. An alternative approach, which does not require as fine a temporal resolution, is to model the relocations. State-space vector autoregression (VAR) models, in which the geographic coordinates of each location are modeled conditionally on the coordinates of the previous location, are natural for this purpose (Dunn and Gipson 1977; Anderson-Sprecher and Ledolter 1991; Worton 1995). Herein we adopt a particular VAR, namely a bivariate Gaussian random walk, as our basic movement model.

As alluded to above, movement of an animal may be influenced by its fidelity to a particular region, commonly called a home range. Home ranges are not only dependent on the length of time over which they are defined, but also are potentially non-stationary over time, so it is important to carefully define the study objectives in order for analyses to be at an appropriate scale. There are many methods for the estimation of home range; for a general review see Worton (1987). The best methods incorporate the idea that the home range is not merely a region but a distribution of the usage of space, or utilization distribution, that ascribes differing probability over the region.

Existing methods for modeling animal movement or estimating the utilization distribution do not directly account for the influence of habitat availability or preference. Note that preference must be quantified on the basis of equal availability among habitats, which rarely occurs. If availability can be determined from a habitat map or some other procedure, then we can estimate relative preference by the relation of use to availability. The resource selection probability function (RSPF) equates the probability of selection (i.e. use) to the availability scaled by preference. The resource selection function (RSF) is defined as the RSPF multiplied by a constant that is generally unknown. See Alldredge et al. (1998) and Manly et al. (2002) for more details and literature reviews on resource selection and RSFs.

One essential step in current methods for calculating resource selection is the definition of available resource units to the animal at a given time. Quite often, availability is defined arbitrarily by the researcher in the context of some predetermined study area in which he/she will conduct the observations. Porter and Church (1987) give an example where conclusions on preference are reversed depending on what is defined as available. Tied up with this issue is the interplay between home range estimation and availability. Some approaches require first using the data to get an estimate of home range, and then using that home range to define availability. Although first estimating home range is certainly more appropriate in the sense that the animal is "deciding" what area is available, it still has flaws. An animal's movement is associated with its need for desirable habitat, so it doesn't really make sense to separate the estimation of where it goes from the estimation of where it prefers to go. Note that, 
in general, telemetry data support the estimation of an animal's usage of space but not the identification of its specific activity (e.g. feeding, sleeping, or mere transit) at any relocation site.

A final issue pertaining to the estimation of an RSF from telemetry data is temporal autocorrelation. Until very recently, the standard technology for obtaining animal relocations was very high frequency (VHF) radio location. With this technology, it was not possible to collect high-frequency location data over long time periods without enormous effort and expense. Sporadic observations generally led to low autocorrelation (barring confounding with circadian behavior patterns), so when calculating resource selection estimates, the assumption of uncorrelated observations required for valid inference was reasonably well satisfied. With the advent of global positioning system (GPS) locators, however, there has been a marked increase in the availability of telemetry data that are highly autocorrelated. Although point estimates of an RSF obtained as if the relocations were independent may be unbiased in the presence of autocorrelation, inferences will be inefficient and variances are likely to be underestimated (Boyce et al. 2002).

All of these issues suggest that the notions of animal movement, utilization distribution, and habitat selection are intertwined, and thus the most satisfactory modeling of any of these notions may result by considering all of them simultaneously. The goal of this article is to develop a model of animal movement that incorporates the notions of home range and habitat selection. More specifically, we will build a model that (a) allows for local movements on spatial and temporal scales that match the temporal resolution of animal relocations, (b) incorporates fidelity to a site or region at a relatively coarse scale, and (c) includes parameters for habitat preference that affect local movements. As the model is too complex for likelihood-based inference to be feasible, we use Bayesian methods to estimate model parameters and perform other inferences of interest. These methods yield in particular estimates of relative preference coefficients for habitat descriptors and a quantification of their uncertainties. We illustrate the methodology using telemetry data on a brown bear in southeast Alaska.

\section{Proposed model}

\subsection{A heuristic movement model}

Let $\mathbf{x}_{t}=\left(x_{1 t}, x_{2 t}\right)^{\prime}$ be the location in two-dimensional space $\mathbf{R}^{2}$ of an animal at time index $t$. Define the fidelity distribution (hereafter we will assume that all distributions are absolutely continuous and described by the associated density function), $f_{f}\left(\mathbf{x}_{t}\right)$, as the timeindependent distribution that governs the animal's overall use of space. In general, there are no restrictions on the form of this distribution. For example, we might define it as a uniform distribution over a specified region for an animal contained inside a relatively small fenced area. Alternatively, we might take it to be bivariate normal if there is a central point around which the animal's movement is concentrated-a den, hiding spot, water hole, etc. It might also be defined as a weighted sum of bivariate normals if there are several such points.

The movement distribution, $f_{m}\left(\mathbf{x}_{t} \mid \mathbf{x}_{t-1}\right)$, is defined, in the absence of site fidelity, conditionally on the animal's previous known location. Again, this distribution can take different forms, but a bivariate normal centered at $\mathbf{x}_{t-1}$ is an obvious choice. We will assume initially that locations are observed at regular time intervals. To obtain the fidelity-weighted 
conditional movement distribution, we simply multiply the fidelity and movement distributions together and re-normalize:

$$
f_{c}\left(\mathbf{x}_{t} \mid \mathbf{x}_{t-1}\right) \propto f_{f}\left(\mathbf{x}_{t}\right) f_{m}\left(\mathbf{x}_{t} \mid \mathbf{x}_{t-1}\right) .
$$

Depending on the form of the distributions, typically this scheme will not only reduce the density for new locations as they get farther from the previous location, but also reduce the density for points that correspond to low density in the fidelity distribution. For this article we assume that the fidelity distribution is bivariate normal with unknown mean vector $\boldsymbol{\mu}$ and positive definite covariance matrix $\boldsymbol{\Sigma}_{f}$. Then

$$
f_{f}\left(\mathbf{x}_{t} \mid \boldsymbol{\mu}, \boldsymbol{\Sigma}_{f}\right) \propto\left|\boldsymbol{\Sigma}_{f}\right|^{-1 / 2} \exp \left[-\frac{1}{2}\left(\mathbf{x}_{t}-\boldsymbol{\mu}\right)^{\prime} \boldsymbol{\Sigma}_{f}^{-1}\left(\mathbf{x}_{t}-\boldsymbol{\mu}\right)\right] .
$$

If we also assume that the movement distribution is bivariate normal, centered at the previous location, then

$$
f_{m}\left(\mathbf{x}_{t} \mid \mathbf{x}_{t-1}, \boldsymbol{\Sigma}_{m}\right) \propto\left|\boldsymbol{\Sigma}_{m}\right|^{-1 / 2} \exp \left[-\frac{1}{2}\left(\mathbf{x}_{t}-\mathbf{x}_{t-1}\right)^{\prime} \boldsymbol{\Sigma}_{m}^{-1}\left(\mathbf{x}_{t}-\mathbf{x}_{t-1}\right)\right]
$$

is the conditional density of the location at time $t$, assuming no fidelity is present. Discounting any effect of fidelity or habitat, there is no compelling reason why an animal would choose to move with more variability in the north-south direction as compared to the east-west direction. For this reason we further assume that $\boldsymbol{\Sigma}_{m}=\sigma_{m}^{2} \mathbf{I}$, an assumption also made by Anderson-Sprecher and Ledolter (1991).

Multiplying these two densities together and re-normalizing, we obtain the fidelityweighted conditional movement density

$$
f_{c}\left(\mathbf{x}_{t} \mid \mathbf{x}_{t-1}, \boldsymbol{\mu}, \boldsymbol{\Sigma}_{f}, \sigma_{m}^{2}\right) \propto\left|\boldsymbol{\Sigma}_{c}\right|^{-1 / 2} \exp \left[-\frac{1}{2}\left(\dot{\mathbf{x}}_{t}-\frac{1}{\sigma_{m}^{2}} \boldsymbol{\Sigma}_{c} \dot{\mathbf{x}}_{t-1}\right)^{\prime} \boldsymbol{\Sigma}_{c}^{-1}\left(\dot{\mathbf{x}}_{t}-\frac{1}{\sigma_{m}^{2}} \boldsymbol{\Sigma}_{c} \dot{\mathbf{x}}_{t-1}\right)\right]
$$

where $\dot{\mathbf{x}}_{t}=\mathbf{x}_{t}-\boldsymbol{\mu}$ and $\boldsymbol{\Sigma}_{c}=\left(\boldsymbol{\Sigma}_{f}^{-1}+\frac{1}{\sigma_{m}^{2}} \mathbf{I}\right)^{-1}$. This is simply another bivariate normal density (see Fig. 1a-c). Note that $f_{c}$, relative to $f_{m}$, has its center shrunk toward $\boldsymbol{\mu}$, and that the size and shape of the equiprobability contours have also changed.

Finally, the likelihood function of all observations save the first is given by

$$
\begin{aligned}
& f_{c}\left(\mathbf{x}_{2}, \ldots, \mathbf{x}_{n} \mid \mathbf{x}_{1}, \boldsymbol{\mu}, \boldsymbol{\Sigma}_{f}, \sigma_{m}^{2}\right) \propto \prod_{t=2}^{n}\left|\boldsymbol{\Sigma}_{c}\right|^{-1 / 2} \\
& \quad \times \exp \left[-\frac{1}{2}\left(\dot{\mathbf{x}}_{t}-\frac{1}{\sigma_{m}^{2}} \boldsymbol{\Sigma}_{c} \dot{\mathbf{x}}_{t-1}\right)^{\prime} \boldsymbol{\Sigma}_{c}^{-1}\left(\dot{\mathbf{x}}_{t}-\frac{1}{\sigma_{m}^{2}} \boldsymbol{\Sigma}_{c} \dot{\mathbf{x}}_{t-1}\right)\right] .
\end{aligned}
$$

Note that we fix the first observed location for simplicity. Because the complexity of the likelihood is greatly reduced, the very minor loss of information is acceptable.

\subsection{Accounting for missing observations}

The current model assumes that observations are made at equally spaced time points, but we can also easily generalize to allow for missing observations. In fact, the model can be extended to handle arbitrary observation times, but here we shall only deal with observation times that are an integer multiple of a constant sampling interval. Note that only the movement portion of the distribution changes when the time interval changes. Because (1) implies that $\left\{\mathbf{x}_{t}\right\}$ is a bivariate Gaussian random walk apart from the influence of fidelity, it can be 
a

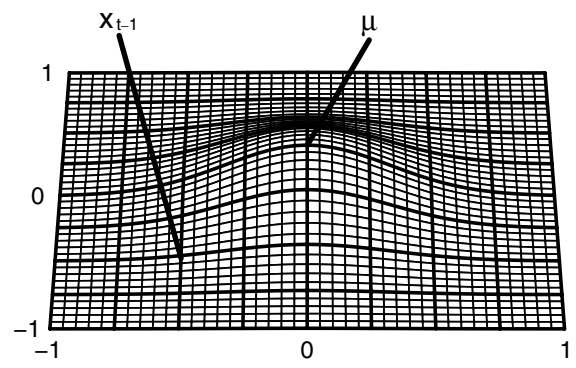

C Fidelity-weighted Conditional Movement

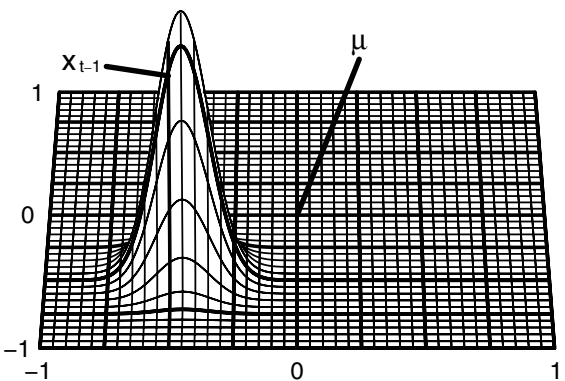

b Movement

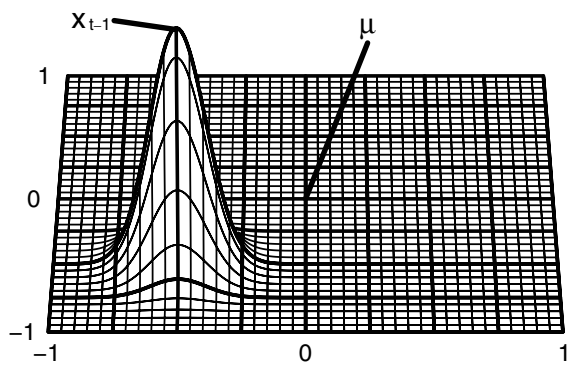

With Habitat Effect

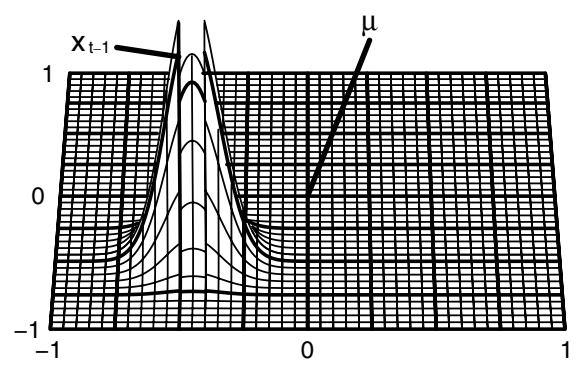

Fig. 1 Depiction of various model components and overall model. Center of attraction is represented by $\boldsymbol{\mu}$, and current location is represented by $\mathbf{x}_{t-1}$

shown that

$$
\mathbf{x}_{t+k-1} \mid \mathbf{x}_{t-1}, \boldsymbol{\Sigma}_{m} \sim \mathrm{N}\left(\mathbf{x}_{t-1}, k \boldsymbol{\Sigma}_{m}\right)
$$

i.e., the covariance matrix grows linearly as time progresses. Let $\Delta_{t}$ represent the integer number of time steps elapsed between $\mathbf{x}_{t}$ and $\mathbf{x}_{t-1}$. We can now generalize the conditional movement density to

$$
f_{m}\left(\mathbf{x}_{t} \mid \mathbf{x}_{t-1}, \sigma_{m}^{2}\right) \propto\left|\Delta_{t} \boldsymbol{\Sigma}_{m}\right|^{-1 / 2} \exp \left[-\frac{1}{2}\left(\mathbf{x}_{t}-\mathbf{x}_{t-1}\right)^{\prime} \frac{1}{\Delta_{t}} \boldsymbol{\Sigma}_{m}^{-1}\left(\mathbf{x}_{t}-\mathbf{x}_{t-1}\right)\right]
$$

and thus the likelihood function is given by

$$
f_{c}\left(\mathbf{x}_{2}, \ldots, \mathbf{x}_{n} \mid \mathbf{x}_{1}, \boldsymbol{\Sigma}_{f}, \sigma_{m}^{2}\right) \propto \prod_{t=2}^{n}\left|\boldsymbol{\Sigma}_{c, t}\right|^{-1 / 2} \exp \left(-\frac{1}{2} \mathbf{a}_{t}^{\prime} \boldsymbol{\Sigma}_{c, t}^{-1} \mathbf{a}_{t}\right)
$$

where $\mathbf{a}_{t}=\dot{\mathbf{x}}_{t}-\frac{1}{\Delta_{t} \sigma_{m}^{2}} \boldsymbol{\Sigma}_{c, t} \dot{\mathbf{x}}_{t-1}$ and $\boldsymbol{\Sigma}_{c, t}=\left(\boldsymbol{\Sigma}_{f}^{-1}+\frac{1}{\Delta_{t} \sigma_{m}^{2}} \mathbf{I}\right)^{-1}$.

\subsection{Reparameterizing the movement model}

The current parameterization suffers from an apparent problem with identifiability. In addition, generating inverse Wishart candidates for $\boldsymbol{\Sigma}_{f}$ and $\boldsymbol{\Sigma}_{m}$ sometimes results in poor convergence properties in an MCMC sampler. Therefore, we propose the following parameterization, which is based on the Cholesky decomposition of $\boldsymbol{\Sigma}_{f}$. For some 


$$
\mathbf{L}=\left(\begin{array}{cc}
1 & 0 \\
r_{1} & r_{2}
\end{array}\right)
$$

and $\eta^{2}>0$, let

$$
\boldsymbol{\Sigma}_{f}=\eta^{2} \mathbf{L} \mathbf{L}^{\prime}=\left(\begin{array}{cc}
\eta^{2} & \eta^{2} r_{1} \\
\eta^{2} r_{1} & \eta^{2}\left(r_{1}^{2}+r_{2}^{2}\right)
\end{array}\right)
$$

This parameterization guarantees a symmetric, positive-definite matrix. If we further restrict $r_{2}>0$ we are guaranteed a unique value for $\mathbf{L}$ (and $\eta^{2}$ ). Now let

$$
\delta^{2}=\left(\frac{1}{\eta^{2}}+\frac{1}{\sigma_{m}^{2}}\right)^{-1}=\frac{\eta^{2} \sigma_{m}^{2}}{\eta^{2}+\sigma_{m}^{2}}, \quad \pi=\frac{\delta^{2}}{\sigma_{m}^{2}}=\frac{\eta^{2}}{\eta^{2}+\sigma_{m}^{2}} .
$$

Note that $0<\pi<1$. We now have

$$
\boldsymbol{\Sigma}_{c, t}=\delta^{2}\left[(1-\pi)\left(\mathbf{L} \mathbf{L}^{\prime}\right)^{-1}+\frac{\pi}{\Delta_{t}} \mathbf{I}\right]^{-1} \quad \text { and } \quad \boldsymbol{\Phi}_{t} \equiv \boldsymbol{\Sigma}_{c, t} \boldsymbol{\Sigma}_{m}^{-1}=\frac{\pi}{\Delta_{t}}\left[(1-\pi)\left(\mathbf{L} \mathbf{L}^{\prime}\right)^{-1}+\frac{\pi}{\Delta_{t}} \mathbf{I}\right]^{-1}
$$

and we may now write the likelihood function as

$$
f_{c}\left(\mathbf{x}_{2}, \ldots, \mathbf{x}_{n} \mid \mathbf{x}_{1}, \boldsymbol{\theta}\right) \propto \prod_{t=2}^{n}\left|\boldsymbol{\Sigma}_{c, t}\right|^{-1 / 2} \exp \left[-\frac{1}{2}\left(\dot{\mathbf{x}}_{t}-\boldsymbol{\Phi}_{t} \dot{\mathbf{x}}_{t-1}\right)^{\prime} \boldsymbol{\Sigma}_{c, t}^{-1}\left(\dot{\mathbf{x}}_{t}-\boldsymbol{\Phi}_{t} \dot{\mathbf{x}}_{t-1}\right)\right] .
$$

where $\boldsymbol{\theta}=\left(\delta^{2}, \pi, r_{1}, r_{2}, \boldsymbol{\mu}^{\prime}\right)^{\prime}$. This parameterization has two major advantages. First, $\delta^{2}$ and $\pi$ have much stronger peaks in their joint likelihood, so the sampler will traverse more of the parameter space and converge faster. Second, we can use a random walk sampler on $r_{1}$ and $r_{2}$ and thus will always draw a valid covariance matrix without having to generate a candidate from an inverse Wishart distribution (Banerjee et al. 2003, pg. 238).

\subsection{Incorporating habitat and other spatial covariates}

The habitat types in the vicinity of an animal may influence where that animal will move. Therefore, we introduce a multiplicative function into the likelihood that depends on the available habitat types at any possible location. Let $\alpha=\left(\alpha_{1}, \ldots, \alpha_{S}\right)^{\prime}$ be some measure of relative preference of the $S$ habitat types. For a single lag-1 observation, we modify the conditional likelihood to be:

$$
f\left(\mathbf{x}_{t} \mid \mathbf{x}_{t-1}, \boldsymbol{\theta}, \boldsymbol{\alpha}\right)=K_{t}\left(\mathbf{x}_{t-1}, \boldsymbol{\theta}, \boldsymbol{\alpha}\right)^{-1} f_{c}\left(\mathbf{x}_{t} \mid \mathbf{x}_{t-1}, \boldsymbol{\theta}\right) \exp \left[\sum_{s=1}^{S} \alpha_{s} I_{s}\left(\mathbf{x}_{t}\right)\right]
$$

where $I_{s}\left(\mathbf{x}_{t}\right)$ is an indicator function that equals 1 if location $\mathbf{x}_{t}$ is in habitat $s$, and equals 0 otherwise, and $K_{t}\left(\mathbf{x}_{t-1}, \boldsymbol{\theta}, \boldsymbol{\alpha}\right)$ is a normalizing function that depends on the parameters, the location at time $t-1$, and the spatial habitat information.

Because we estimate the parameters of $f$ using Bayesian methods involving importance ratios, it is necessary to evaluate $K_{t}\left(\mathbf{x}_{t-1}, \boldsymbol{\theta}, \boldsymbol{\alpha}\right)$. To this end, let $\mathbf{R}_{s}^{2}$ be the collection of regions in space that are habitat type $s(s=1,2, \ldots, S)$, and let $\operatorname{Pr}_{t}(s)$ be the probability (conditional on $\mathbf{x}_{t-1}$ and $\boldsymbol{\theta}$ ) that the animal's location at time $t$ falls within habitat type $s$ in the absence of habitat preference. Then 


$$
\begin{aligned}
1 & =\iint_{\mathbf{R}^{2}} f\left(\mathbf{x}_{t} \mid \mathbf{x}_{t-1}, \boldsymbol{\theta}, \boldsymbol{\alpha}\right) d \mathbf{x}_{t} \\
& =\sum_{s=1}^{S} \iint_{\mathbf{R}_{s}^{2}} K_{t}\left(\mathbf{x}_{t-1}, \boldsymbol{\theta}, \boldsymbol{\alpha}\right)^{-1} f_{c}\left(\mathbf{x}_{t} \mid \mathbf{x}_{t-1}, \boldsymbol{\theta}\right) \exp \left(\alpha_{s}\right) d \mathbf{x}_{t} \\
& =K_{t}\left(\mathbf{x}_{t-1}, \boldsymbol{\theta}, \boldsymbol{\alpha}\right)^{-1} \sum_{s=1}^{S}\left[\exp \left(\alpha_{s}\right) \operatorname{Pr}_{t}(s)\right]
\end{aligned}
$$

It follows that

$$
K_{t}\left(\mathbf{x}_{t-1}, \boldsymbol{\theta}, \boldsymbol{\alpha}\right)=\sum_{s=1}^{S}\left[\exp \left(\alpha_{s}\right) \operatorname{Pr}_{t}(s)\right]
$$

so we now alter the likelihood to be:

$$
\begin{aligned}
f\left(\mathbf{x}_{2}, \ldots, \mathbf{x}_{n} \mid \mathbf{x}_{1}, \boldsymbol{\theta}, \boldsymbol{\alpha}\right) \propto \prod_{t=2}^{n} & K_{t}\left(\mathbf{x}_{t-1}, \boldsymbol{\theta}, \boldsymbol{\alpha}\right)^{-1}\left|\boldsymbol{\Sigma}_{c, t}\right|^{-1 / 2} \\
& \times \exp \left[-\frac{1}{2}\left(\dot{\mathbf{x}}_{t}-\boldsymbol{\Phi}_{t} \dot{\mathbf{x}}_{t-1}\right)^{\prime} \boldsymbol{\Sigma}_{c, t}^{-1}\left(\dot{\mathbf{x}}_{t}-\boldsymbol{\Phi}_{t} \dot{\mathbf{x}}_{t-1}\right)\right] \\
& \times \exp \left[\sum_{s=1}^{S} \alpha_{S} I_{S}\left(\mathbf{x}_{t}\right)\right] .
\end{aligned}
$$

Figure $1 \mathrm{~d}$ shows the effects of introducing a habitat covariate. The non-shaded region, $-0.5<$ $x<-0.4$, has lower preference and thus the likelihood is lower in that region. One nice feature of this particular model is that we can exclude habitat types that we know are not used (e.g. water, rock formations, etc.) by setting the associated $\alpha$ to $-\infty$.

The $\alpha$ 's, however, are not estimable without further restrictions. For some arbitrary constant vector $\mathbf{q}=q \mathbf{1}_{S}$, where $\mathbf{1}_{S}$ is a vector of length $S$ containing all ones, it can be shown that

$$
K_{t}\left(\mathbf{x}_{t-1}, \boldsymbol{\theta}, \boldsymbol{\alpha}+\mathbf{q}\right)^{-1} \exp \left[\sum_{s=1}^{S}\left(\alpha_{S}+q\right) I_{S}\left(\mathbf{x}_{t}\right)\right]=K_{t}\left(\mathbf{x}_{t-1}, \boldsymbol{\theta}, \boldsymbol{\alpha}\right)^{-1} \exp \left[\sum_{s=1}^{S} \alpha_{S} I_{S}\left(\mathbf{x}_{t}\right)\right] .
$$

Therefore, we propose to set one of the $\alpha$ 's equal to zero, which will in effect treat that habitat as a baseline to which all other habitat types will be compared; that is, a negative $\alpha$ will reflect less preference and a positive $\alpha$ will reflect greater preference as compared to the baseline habitat type.

In order to render this method for estimating habitat preference parameters comparable with existing methods, we need to standardize by forming $\exp \left(\alpha_{1}\right) / \sum_{s=1}^{S} \exp \left(\alpha_{s}\right), \ldots$, $\exp \left(\alpha_{S}\right) / \sum_{s=1}^{S} \exp \left(\alpha_{S}\right)$. These are analogous to the standardized resource selection coefficients as described in Manly et al. (2002) because preference is equivalent to selection under equal availability.

We need not limit our consideration to categorical spatial covariates. It is also possible to model preference for continuous covariates such as elevation, distance to water, etc. To illustrate, consider a single lag-1 observation with a single continuous spatial covariate. For this observation we can write the conditional likelihood as

$$
f\left(\mathbf{x}_{t} \mid \mathbf{x}_{t-1}, \boldsymbol{\theta}, \boldsymbol{\alpha}\right)=K_{t}\left(\mathbf{x}_{t-1}, \boldsymbol{\theta}, \boldsymbol{\alpha}\right)^{-1} f_{c}\left(\mathbf{x}_{t} \mid \mathbf{x}_{t-1}, \boldsymbol{\theta}\right) \exp \left[\alpha g\left(\mathbf{x}_{t}\right)\right]
$$


where $g\left(\mathbf{x}_{t}\right)$ is the value of the continuous spatial covariate at $\mathbf{x}_{t}$. Unfortunately, upon observing that

$$
K_{t}\left(\mathbf{x}_{t-1}, \boldsymbol{\theta}, \boldsymbol{\alpha}\right)=\iint_{\mathbf{R}^{2}} f_{c}\left(\mathbf{x}_{t} \mid \mathbf{x}_{t-1}, \boldsymbol{\theta}\right) \exp \left[\alpha g\left(\mathbf{x}_{t}\right)\right] d \mathbf{x}_{t},
$$

we see that it is not practical to evaluate $K_{t}\left(\mathbf{x}_{t-1}, \boldsymbol{\theta}, \boldsymbol{\alpha}\right)$ in this case. Therefore, we choose to simplify this problem by instead looking at a discrete grid of continuous covariate values. This does not necessarily result in a loss of information because continuous spatial covariates are often stored in a GIS as rasters (grids). We now have

$$
K_{t}\left(\mathbf{x}_{t-1}, \boldsymbol{\theta}, \boldsymbol{\alpha}\right)=\sum_{d=1}^{D} \iint_{\mathbf{R}_{d}^{2}} f_{c}\left(\mathbf{x}_{t} \mid \mathbf{x}_{t-1}, \boldsymbol{\theta}\right) \exp \left[\alpha g_{d}\left(\mathbf{x}_{t}\right)\right] d \mathbf{x}_{t}=\operatorname{Pr}_{t}\left(g_{d}\right) \exp \left(\alpha g_{d}\right)
$$

where $g_{d}, d=1, \ldots, D$ is the collection of values the spatial covariate can take on, $\mathbf{R}_{d}^{2}$ is the region where $g\left(\mathbf{x}_{t}\right)=g_{d}$, and $\operatorname{Pr}_{t}\left(g_{d}\right)$ is the probability that $\mathbf{x}_{t}$ falls in $\mathbf{R}_{d}^{2}$ in the absence of habitat preference. The identifiability problem that exists in the categorical case does not occur in this case because we are now dealing with a single alpha, which is much more like a regression coefficient.

The $g_{d}$ can be standardized for ease of interpretation. For a case (such as our brown bear example) where we are modeling distance to some feature, suppose the $g_{d}$ occur at regular increments such that $c=g_{d}-g_{d-1}$ for all $d$, and thus $g_{d}=g_{1}+(d-1) c$. Then we have

$$
\begin{aligned}
f\left(\mathbf{x}_{t} \mid \mathbf{x}_{t-1}, \boldsymbol{\theta}, \boldsymbol{\alpha}\right) & =\frac{f_{c}\left(\mathbf{x}_{t} \mid \mathbf{x}_{t-1}, \boldsymbol{\theta}\right) \exp \left\{\alpha\left[g_{1}+\left(d_{\mathbf{x}_{t}}-1\right) c\right]\right\}}{\sum_{d=1}^{D} \exp \left\{\alpha\left[g_{1}+(d-1) c\right]\right\} \operatorname{Pr}_{t}\left(g_{d}\right)} \\
& =\frac{\left.f_{c}\left(\mathbf{x}_{t} \mid \mathbf{x}_{t-1}, \boldsymbol{\theta}\right) \exp \left\{\alpha c\left(d_{\mathbf{x}_{t}}-1\right)\right]\right\} \exp \left(\alpha g_{1}\right)}{\sum_{d=1}^{D} \exp \{\alpha c(d-1)\} \exp \left(\alpha g_{1}\right) \operatorname{Pr}_{t}\left(g_{d}\right)} \\
& =\frac{f_{c}\left(\mathbf{x}_{t} \mid \mathbf{x}_{t-1}, \boldsymbol{\theta}\right) \exp \left[(\alpha c) d_{\mathbf{x}_{t}}^{*}\right]}{\sum_{d^{*}=0}^{D-1} \exp \left(\alpha c d^{*}\right) \operatorname{Pr}_{t}\left(g_{d^{*}}\right)}
\end{aligned}
$$

where $d_{\mathbf{x}_{t}}$ is the distance class of $\mathbf{x}_{t}, d_{\mathbf{x}_{t}}^{*}=d_{\mathbf{x}_{t}}-1$, and $d^{*}=d-1$. We see that $\alpha$ is scaled by $c$ as we would expect, and we can regard this as a new parameter, $\alpha^{*}=\alpha c$. Our distance classes are now the nonnegative integers, so using the lowest value as the base (i.e. the likelihood is unchanged for this class), we have an approximate $\alpha \%$ change in the likelihood for every pixel we move away from this base. For example, if the data are on a $50 \mathrm{~m}$ grid, $\alpha=-0.1$ would correspond to a $10 \%$ drop in the likelihood for every $50 \mathrm{~m}$ away from the area that corresponds to this base value.

\subsection{Estimation of parameters}

When spatial covariates are included it is apparent from $K_{t}\left(\mathbf{x}_{t-1}, \boldsymbol{\theta}, \boldsymbol{\alpha}\right)$ that maximum likelihood estimators will be extremely difficult to calculate. From (2) it is clear that, for each location at time indices $t=1, \ldots, T$ we must evaluate $\operatorname{Pr}_{t}(s)$ for each of the $S$ habitat types. Because the region may have a very complex arrangement of habitats, this probability statement will likewise be very complex in all but the most trivial of cases.

For Bayesian analysis we only need to be able to evaluate the likelihood using proposed parameter values. This is by no means trivial, but at least it is tractable. We utilize a Metropolis-Hastings algorithm written in R to obtain parameter estimates. Non-informative, improper flat priors are placed on all parameters, except for the habitat selection parameters for which we take the prior to be $\mathrm{N}(\mathbf{0}, 100 \mathrm{I})$. This restricts the magnitude of the $\alpha$ 's and is particularly 
important when a certain habitat is rarely chosen or not chosen at all. In this case, there is not enough information in the data to overcome a very strong $\alpha$ coefficient. Candidates are generated using an appropriately tuned random walk sampler. Posterior means may be regarded as point estimates of model parameters, and their uncertainty is characterized by, say, the 2.5th and 97.5th quantiles of the marginal posterior distributions.

As we iterate through the sampler and new values of $\delta^{2}, \pi, r_{1}, r_{2}, \boldsymbol{\mu}$, and $\boldsymbol{\alpha}$ are proposed, it is necessary to compute $K_{t}\left(\mathbf{x}_{t-1}, \boldsymbol{\theta}, \boldsymbol{\alpha}\right)$ for each observation $\mathbf{x}_{t}$. The greatest difficulty with this lies in calculating the probability of moving to a region of each habitat type, $\operatorname{Pr}_{t}(s)$. Currently, this is done by numerically integrating over a pre-specified grid. Although this can be estimated very closely using closed-form methods to actually calculate $\operatorname{Pr}_{t}(s)$, it is very time consuming and therefore impractical. We have found that evaluating the underlying constituent likelihoods on a grid, multiplying (elementwise) the respective grids, and then summing over the grid achieves reasonable accuracy with very fast speed.

\section{Brown bear example}

For a study of habitat preference for brown bears in Southeast Alaska, relocations of a single female bear with a GPS collar were attempted every 20 min over an 11 day period during the salmon runs in October 2001. Due to problems with early generation antenna design and satellite acquisition, a large number of relocations were not acquired. Overall, 157 observations were recorded during the study period and the largest time gap for consecutive observations was 28 intervals. We assume that the missing observations are missing at random, although such an assumption should be made carefully.

Habitat data is in the form of a raster with $50 \mathrm{~m}$ by $50 \mathrm{~m}$ pixels and is shown in Fig. 2. The habitats are tidal flat, nonforest, clearcut, scrub forest, small forest, medium forest and large forest. Because the tidal flats have at most a small probability of availability for any relocation, there is no information from the data regarding the preference for this habitat so it is not included in the analysis. Nonforest, however, is slightly different; the bear never was relocated in nonforest, but this habitat is available with a reasonable probability for some relocations, so there is enough information, albeit minimal, to estimate preference.

We include a second spatially located habitat variable, namely, distance to stream, which we discretize to the same $50 \mathrm{~m}$ grid, so that it becomes an ordered categorical variable. The corresponding parameter will be a function of the rate of decrease in attraction to the streams. We restrict this parameter to be negative because we know that bears are attracted to the salmon streams and do not actively avoid them. For simplicity, the distance to stream categories are rescaled to nonnegative integers as outlined in Sect. 2.4.

The MCMC sampler is run for 50,000 iterations after burn-in, meeting appropriate convergence criteria. Table 1 shows selected results for the posterior distributions of the standardized habitat preference coefficients. Note that tidal flats are not included in the standardization. Large forest is shown to be strongly preferred, medium forest less so, and clearcut, scrub forest and small forest are all statistically indistinguishable. Nonforest is strongly avoided. We also see an effect for distance to stream which corresponds to roughly a $1 \%$ additional drop in the likelihood for every $50 \mathrm{~m}$ distance from the stream.

The inferences available from this model are not restricted solely to the parameters presented. One of the benefits of such a Bayesian analysis is that once we have estimated the posterior distribution, we can then sample any function of the parameters and estimate its distribution. For example, we can use the model output to get a surface representing the utilization distribution (UD) of the animal. By sampling from this distribution, we can estimate 


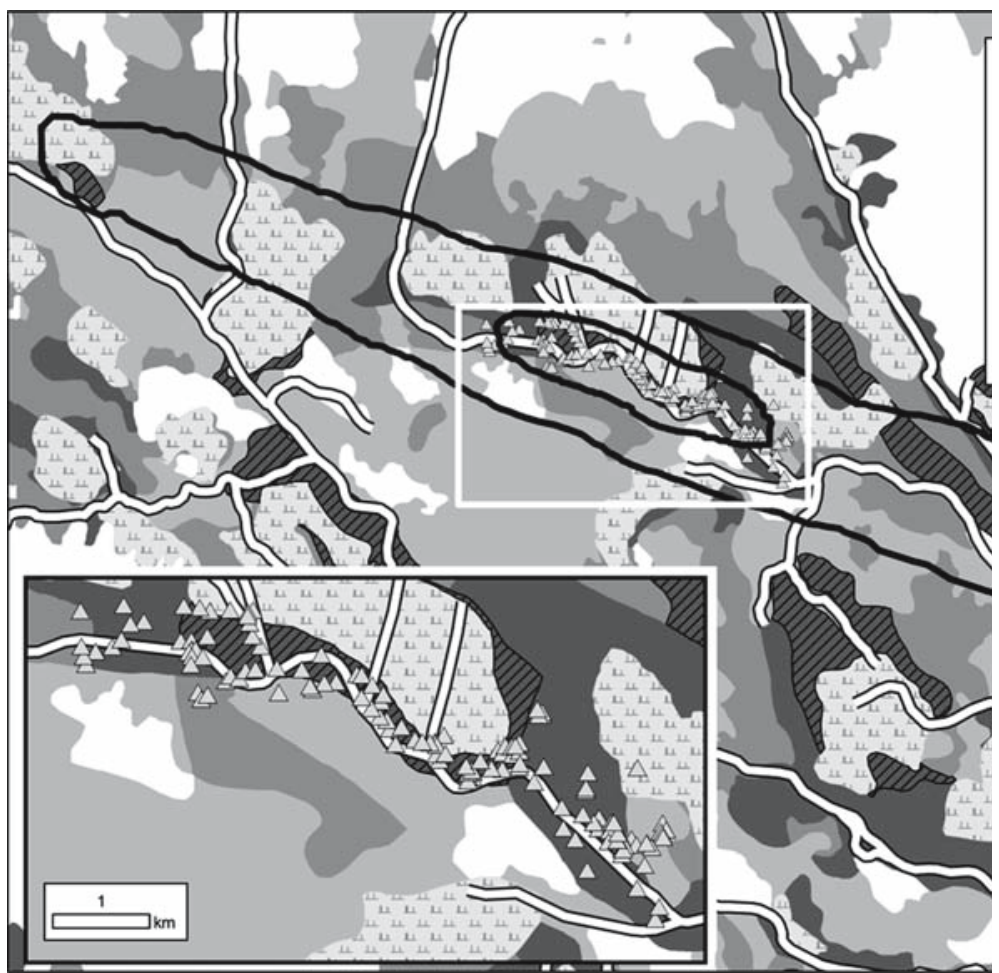

Fig. 2 Habitat map showing bear relocations and UD confidence bands for the brown bear data

Table 1 Posterior distributions of standardized preference coefficients for brown bear data

\begin{tabular}{lrrr}
\hline Parameter & 2.5\% Point & Mean & $97.5 \%$ Point \\
\hline Distance from stream & -0.0398 & -0.0105 & -0.0003 \\
Nonforest & 0.0001 & 0.0115 & 0.0626 \\
Clearcut & 0.0566 & 0.1116 & 0.1886 \\
Scrub forest & 0.0627 & 0.1055 & 0.1617 \\
Small forest & 0.0653 & 0.1582 & 0.2939 \\
Medium forest & 0.1665 & 0.2300 & 0.3051 \\
Large forest & 0.2772 & 0.3832 & 0.4853 \\
\hline
\end{tabular}

the $95 \%$ contour of the UD, and construct a confidence band for this contour as well, thus giving us an idea of the level of uncertainty in the estimate.

Figure 2 includes an example of such a construction. 100 sets of parameters are drawn from the MCMC chain (post burn-in). For each set of parameters, simulated data are generated and the $95 \%$ quantile contour of the UD is calculated using a kernel smoother. The larger contour represents the region that contains points included in at least $5 \%$ of the 100 quantile contours. In some sense this is an outer bound for all $95 \%$ quantile contours: we are relatively certain that any $95 \%$ quantile contour would be contained within this region. Likewise, the smaller contour contains the points included in at least $95 \%$ of the quantile contours. This is similarly regarded as an inner bound for the $95 \%$ quantile contours and we are relatively certain that every contour will contain these points. 
We see that these contour bounds are not perfectly elliptical and are somewhat altered by the various habitats. One might expect there to be a more noticeable habitat effect, but these regions are intersections and do not reflect the variability of the individual $95 \%$ quantile contour replications. Also, the outer contour appears to have an extent much larger than that implied by the data. This can be explained by the fact that we have high autocorrelation and a relatively short time frame for the data. The simulated data used for the quantile contours has a much longer time span and thus has the opportunity to reach regions not observed in the real data.

There may also be some confounding between the estimated fidelity covariance matrix and the habitat effects. Because the locations are contained in a rather linear subregion and because the habitats also exhibit considerable linearity within this subregion, the model may not be able to clearly distinguish between a strongly elliptical fidelity covariance and a strong habitat effect.

As the number of observed locations per animal increase, this estimation procedure quickly becomes computationally intensive, although it could greatly benefit from a parallel computing framework. As the dimensionality of the problem increases (i.e. more distinct habitat categories), we have also seen a sensitivity to how candidates of the various habitat parameters are generated. This generally results in higher autocorrelation in the resulting chain and longer runs required for convergence and estimation. We have noted some boundary issues with the estimation of $\pi$ in certain data with extremely high temporal autocorrelation. It is unclear whether this is an artifact of the parameterization or the estimation procedure and is under further investigation. Future developments include a hierarchical multiple-animal model allowing for the estimation of selection at both a population and individual level. An example of the R code used for this estimation can be found at ftp://ftpr3.adfg.state.ak.us/MISC/amchrist/EES article/.

Acknowledgements Financial support for this work was provided by the Federal Aid in Wildlife Restoration to the Alaska Department of Fish and Game.

\section{References}

Alldredge JR, Thomas DL, McDonald LL (1998) Survey and comparison of methods for study of resource selection. J Agric Biolo Environ Stat 3:237-253

Anderson-Sprecher R, Ledolter J (1991) State-space analysis of wildlife telemetry data. J Am Stati Associ 86:596-602

Banerjee S, Carlin BP, Gelfand AE (2003) Hierarchical modeling and analysis for spatial data. Chapman \& Hall/CRC, Boca Raton

Boyce MS, Vernier PR, Nielsen SE, Schmiegelow FKA (2002) Evaluating resource selection functions. Ecol Modell 157:281-300

Brillinger DR (1997) A particle migrating randomly on a sphere. J Theor Probab 10:429-443

Brillinger DR, Preisler HK, Ager AA, Kie JG, Stewart BS (2002) Employing stochastic differential equations to model wildlife motion. Bull Braz Math Soc 33:385-408

Brillinger DR, Preisler HK, Ager AA, Kie JG (2004) An exploratory data analysis (EDA) of the paths of moving animals. J Stat Plan Inference 122:43-63

Dunn JE, Gipson PS (1977) Analysis of radio telemetry data in studies of home range. Biometrics 33:85-101

Manly BFJ, McDonald LL, Thomas DL, McDonald TL, Erickson WP (2002) Resource selection by animals: statistical design and analysis for field studies 2nd edn., Kluwer, Dordrecht

Porter WF, Church KE (1987) Effects of environmental pattern on habitat preference analysis. J Wildl Manage 51:681-685

Worton BJ (1987) A review of models of home range for animal movement. Ecol Modell 38:277-298

Worton BJ (1995) Modelling radio-tracking data. Environ Ecolo Stat 2:15-23 


\section{Author biographies}

Aaron Christ is a biometrician for the Alaska Department of Fish and Game, Division of Wildlife Conservation in Anchorage, Alaska. He received his Ph.D. in Statistics from the University of Iowa in 2003.

Jay Ver Hoef completed most of this work as a biometrician for the Wildlife Conservation Division of the Alaska Department of Fish and Game. He is currently a statistician with the National Marine Mammal Laboratory in Seattle, where he serves as a statistical consultant and continues his statistical research interests. $\mathrm{He}$ is also an adjunct professor of statistics with the Mathematics Department of the University of Alaska, Fairbanks, and a Fellow of the American Statistical Association. He received his B.S. in botany from Colorado State University, his M.S. in botany from the University of Alaska, Fairbanks, and his Ph.D., a co-major in statistics and EEB (ecology and evolutionary biology), from Iowa State University.

Dale L. Zimmerman is Professor of Statistics in the Department of Statistics and Actuarial Science at the University of Iowa, and a Fellow of the American Statistical Association. He received B.S. degrees in Biometry and Animal Ecology from Iowa State University, an M.S. in Statistics from the University of Minnesota, and his Ph.D. in Statistics from Iowa State University. His research interests include spatial statistics, environmental statistics, longitudinal data analysis, multivariate analysis, and mixed linear models. 